\title{
Dosage du glyphosate par HPLC après extraction et dérivation à l'O-phthaldialdehyde (OPA)
}

\author{
Gains Kouakou KPAN KPAN ${ }^{1 *}$, Ardjouma DEMBELE ${ }^{2}$, \\ Lazare Brou $\mathrm{YAO}^{2}$ et Seydou TIHO ${ }^{1}$
}

\author{
${ }^{1}$ Laboratoire de Biologie et Cytologie Animale, UFR des Sciences de la Nature, \\ Université Nangui Abrogoua, 02 BP 801 Abidjan 02, Côte d'Ivoire. \\ ${ }^{2}$ Laboratoire National d'Appui au Développement Agricole (LANADA), Laboratoire Central \\ d'Agrochimie et d'Ecotoxicologie (LCAE) 04 BP 612 Abidjan 04, Côte d'Ivoire. \\ *Auteur correspondant ; E-mail : oraclegains@gmail.com ; Tel. : (+225) 47663894
}

\section{RESUME}

Le glyphosate, premier herbicide utilisé au monde est une molécule difficile à quantifier par la chromatographie en phase liquide à haute performance (HPLC), eu égard à l'absence de chromophore dans sa structure. La chimie analytique est donc à la recherche perpétuelle de méthodes de détermination du glyphosate par HPLC. Ce travail avait pour but de développer une méthode de quantification du glyphosate du sol par

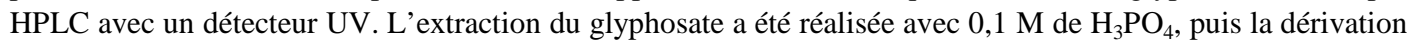
a été faite avec du O-phthaldialdehyde -2-mercaptoéthanol et la détection a été réalisée à $205 \mathrm{~nm}$. Le taux de recouvrement a été de $88,16 \%$, la limite de détection (LD) a valu $0,009 \mathrm{mg} / \mathrm{kg}$ et la limite de quantification (LQ) a été de $0,027 \mathrm{mg} / \mathrm{kg}$. Les coefficients de variation de la répétabilité et de la reproductibilité ont été respectivement $1,1 \%$ et $2,9 \%$. Cette méthode mérite une attention particulière car sa LQ est inférieure aux plus sévères limites maximales de résidus du glyphosate du Codex Alimentarius $(0,05 \mathrm{mg} / \mathrm{kg})$ et de l'Agence Américaine pour la Protection Environnementale $(0,1 \mathrm{mg} / \mathrm{kg})$. En outre, le temps de rétention $(4,568 \mathrm{~min})$ est court et permet par conséquent une économie de solvant.

(C) 2015 International Formulae Group. All rights reserved.

Mots clés : Glyphosate, HPLC, O-phthaldialdehyde, 2-mercaptoéthanol, sol.

\section{INTRODUCTION}

Le glyphosate est un herbicide systémique non sélectif, destiné à combattre les mauvaises herbes en pré-levé et post-levé. Il inhibe l'enzyme 5-enolpyruvyl-shikimate-3phosphate synthétase (EPSPS), spécifique aux plantes et impliquée dans la synthèse des acides aminés (phénylalanine, tyrosine et tryptophane) indispensables au métabolisme de la plante (Schronbrunn, 2001; Duke et Powles, 2008). Sa tolérance pour les animaux, son action effective sur la plupart des adventices et surtout la mise au point de cultures résistantes au glyphosate, font de cette molécule, l'herbicide le plus utilisé au monde (Owen et Zelayan, 2005 ; Li et al., 2007). Son usage s'étend à plusieurs domaines notamment l'agriculture, la voirie, le domaine public, la forestérie et les résidences.

L'usage très répandu de cet herbicide, nécessite la mise au point de stratégies de monitoring qui permettront d'évaluer le 
niveau de contamination des denrées alimentaires et des matrices environnementales par cette molécule afin d'éviter les problèmes environnementaux et de santé publique plausibles.

Plusieurs méthodes, en l'occurrence la chromatographie en phase gazeuse, la chromatographie en phase gazeuse couplée à un spectromètre de masse (GC-MS), la chromatographie en phase liquide, la chromatographie en phase liquide couplée à un spectromètre de masse (LC-MS) ; permettent de quantifier le glyphosate et ses métabolites dans les matrices environnementales (Khrolenko et Wieczorek, 2005 ; Qian et al., 2009).

En dépit de la pluralité des méthodes de dosage du glyphosate, cette molécule reste l'une des plus difficiles à étudier en chimie analytique car elle fait appel à une dérivation, eu égard à l'absence de chromophore dans sa structure (Chiu et al., 2008; Druart et al., 2011). Le O-phthaldialdehyde (OPA), est l'un des réactifs couramment utilisés pour la dérivatisation du glyphosate et de l'acide aminomethylphosphonique

(AMPA). Cependant, dans la plupart des méthodes qui ont recours à l'OPA, la dérivation est post colonne et le dérivé formé est détecté en fluorescence. La détection à l'ultra violet (UV) du complexe glyphosate - OPA est rarement évoquée dans la littérature.

Ce travail avait pour but de mettre au point une méthode de détermination du glyphosate du sol par dérivatisation pré colonne à l'aide de l'OPA et du 2mercaptoéthanol suivie d'une détection à l'UV.

\section{MATERIEL ET METHODES}

Réactifs et solvants

Des étalons de glyphosate $(98 \%$ de pureté), acquis auprès de Dr. Ehrenstorfer $\mathrm{GmbH}$, ont servi à préparer les solutions d'étalonnage. Ces étalons dont la date d'expiration est le 09 /2018 ont été soigneusement conservés à $20 \pm 4{ }^{\circ} \mathrm{C}$ au moyen d'un réfrigérateur COOLTECH (SUMSUNG).

Les réactifs de dérivation à savoir le $\mathrm{O}$ phthaldialdehyde (OPA) de pureté $\geq 99 \%$ et le 2-mercaptoéthanol de pureté $\geq 99 \%$ ont été acquis auprès de SIGMA-ALDRICH et conservés à $4{ }^{\circ} \mathrm{C}$ dans un réfrigérateur EVERmed.

De l'acide phosphorique à $0,1 \quad \mathrm{M}$ (SIGMA-ALDRICH), du tétraborate de sodium (MERCK), du méthanol de grade HPLC (HIPERSOV CHROMMANORM) et de l'eau bidistillée ont été également utilisés pour l'extraction et le dosage du glyphosate.

\section{Appareillage}

Une balance électronique (SATORIUS) de précision $\pm 0,001 \mathrm{~g}$ a permis de peser les échantillons de sol et les réactifs. Un tamis électronique (RETSCH) avec des pores de diamètre allant de $2 \mathrm{~mm}$ à $62 \mu \mathrm{m}$ a servi à réaliser la granulométrie du sol. Le séchage des échantillons de sol a été effectué par le biais d'une étuve (BINDER). Le $\mathrm{pH}$ du sol a été mesuré au moyen d'un multimètre (HACH, HQ40d) tandis que la conductivité a été déterminée à l'aide d'un conductimètre (HANNA). Pour concentrer les solutions extraites des échantillons de sol, un évaporateur rotatif (BUCHI) muni d'un contrôleur de pression (V-880), d'un Rotavapor (R-215), d'un bain marie (B-491) et d'une pompe à vide (V-700) ont été utilisés. Une centrifugeuse Universal 320R $(\mathrm{HETTICH})$ et un four à moufle (NABERTHERM) ont servi respectivement à la centrifugation et à l'incinération des échantillons de sol. Une chaîne de chromatographie en phase liquide à haute performance (SHIMADZU) composée d'un réservoir (TRAY), d'un dégazeur (DGU20A5), d'un échantillonneur automatique (SIL-20A), d'une pompe (LC-20AT), d'un four de type (CTO-20A) et d'un détecteur UV/VIS (SPD-20A) a été utilisée pour la quantification du glyphosate. L'acquisition des données a été faite à l'aide d'un ordinateur muni du logiciel LC solution. 


\section{Préparation des échantillons}

Les échantillons de sol ont été prélevés sur un terrain de jachère d'environ 25 ans, situé dans la localité d'Azaguié se trouvant à environ 30 kilomètres d'Abidjan (Côte d' Ivoire). Les échantillons de sol ont été prélevés à la profondeur de $0-5 \mathrm{~cm}$. Ces échantillons ont été ensachés dans des sacs stomacher puis transportés au Laboratoire Central d'Agrochimie et d'Ecotoxicologie où ils ont été conservés à $4{ }^{\circ} \mathrm{C}$ avant les analyses. La masse de chaque échantillon est de $200 \mathrm{~g}$.

\section{Détermination des propriétés physicochimiques du sol pH}

La méthode de détermination du $\mathrm{pH}$ est inspirée de la méthode de l'extrait dilué (Montoroi, 1997) et du protocole de détermination du $\mathrm{pH}$ élaboré par le Centre d'Expertise en Analyse Environnementale du Québec (MA.100-pH 1.1, version 2003).

\section{Conductivité}

La mesure de la conductivité des échantillons de sol a été opérée conformément aux recommandations de Montoroi (1997). Les échantillons ont été séchés à l'air libre pendant 4 jours, puis tamisés au moyen d'un tamis de $2 \mathrm{~mm}$ de diamètre. Vingt grammes (20g) de sol tamisés ont été ensuite transférés dans un bocal en plastique, puis $100 \mathrm{ml}$ d'eau bidistillée y ont été ajoutés (rapport 1/5). Le mélange a été agité à l'aide d'un agitateur (Vortex) pendant 2 minutes, puis laissé au repos pendant environ 30 minutes. La conductivité a été mesurée à l'aide d'un conductimètre préalablement étalonné, en plongeant l'électrode de l'appareil dans le surnageant de la solution de sol. L'électrode $\mathrm{du}$ conductimètre a été rincée à l'eau bidistillée chaque fois que l'on a changé d'échantillon.

\section{Teneur en matière organique}

La teneur en matière organique des échantillons de sol a été déterminée par la méthode de la perte au feu (PAF) (CEAEQ, 2003 ; Hasine et al., 2008). Une masse de $5 \mathrm{~g}$ de sol séché à $105{ }^{\circ} \mathrm{C}$ à l'étuve pendant 24 heures, a été incinérée à $375^{\circ} \mathrm{C}$ dans un four à moufle pendant 16 heures, puis la teneur en matière organique (MO) a été déduite par gravimétrie suivant l'équation :

$$
\% \text { MO }=\frac{(\text { massede sol sec }- \text { masse de cenare }) \times 100}{\text { masse de sol sec }}
$$

\section{Granulométrie}

La granulométrie de la fraction fine du sol a été effectuée par tamisage. L'échantillon de sol a été séché à l'air libre durant 48 heures, puis il a été débarrassé des débris végétaux et animaux. L'échantillon trié a été chauffé à l'étuve pendant 24 heures à $105{ }^{\circ} \mathrm{C}$ afin d'éliminer l'eau résiduelle. Après le séchage à l'étuve, l'échantillon est passé sur un tamis de $2 \mathrm{~mm}$ de diamètre, puis $25 \mathrm{~g}$ de la fraction de sol dont le diamètre est inférieur à $2 \mathrm{~mm}$ ont été tamisés avec une superposition de tamis de diamètre décroissant (125 $\mu \mathrm{m}-62$ $\mu \mathrm{m})$.

La fraction du sol dont le diamètre $\geq 125 \mu \mathrm{m}$ a été qualifiée de sable grossier, celle dont le diamètre appartient l'intervalle < $125 \mu \mathrm{m} \geq 62 \mu \mathrm{m}$ a été qualifiée de sable fin tandis que la fraction du sol dont le diamètre est $<62 \mu \mathrm{m}$ a été assignée à proportion des argiles et du limon.

\section{Dosage du glyphosate \\ Extraction du glyphosate du sol}

Chaque échantillon de sol (200 g) a été séché à l'air libre, à l'abri du soleil durant 4 à 5 jours. Après séchage, l'échantillon a été trié, puis broyé finement au mortier. A $25 \mathrm{~g} \mathrm{du}$ broyat, $100 \mathrm{ml}$ d'une solution d'acide phosphorique à $0,1 \mathrm{M}$ (dans de l'eau bidistillée) ont été ajoutés. Cette mixture a été homogénéisée sur un agitateur pendant 5 heures. Ensuite, le mélange a été centrifugé à $5000 \mathrm{rpm}$ pendant 20 minutes, puis le surnageant a été filtré sur du Whatman $\mathrm{n}^{\circ} 1$ et concentré à $10 \mathrm{ml}$ à l'aide d'un évaporateur rotatif. L'extrait concentré $(10 \mathrm{ml})$ a été filtré sur un filtre à disque de $0,2 \mu \mathrm{m}$ de diamètre afin de procéder à la dérivation. 
Préparation des solutions étalons de glyphosate

Les étalons de glyphosate ont été préparés par dissolution de cristaux purs de glyphosate (forme acide) dans l'eau bidistillée. Ainsi, quatre solutions étalons de concentrations $0,076, \quad 0,76,7,6$ et $15,2 \mu \mathrm{g} / \mathrm{l}$ ont été obtenues.

\section{Préparation de la solution de dérivation}

La solution de dérivation est constituée d'un mélange de O-phthaldialdehyde (OPA) et de 2-mercaptoéthanol (2-MCE). La réaction qui aboutit à la formation du complexe OPA2MCE a lieu dans un milieu basique $(\mathrm{pH}=9)$. Pour créer ce milieu, le tampon de tétraborate de sodium a été utilisé. Ce tampon a été préparé par la dissolution de $3,8 \mathrm{~g}$ de tétraborate dans $100 \mathrm{ml}$ d'eau bidistillée. La solution obtenue a été filtrée sur du papier filtre Whatman afin d'éliminer d'éventuels cristaux qui y seraient présents. Ensuite, 120 mg d'OPA ont été dissouts dans $3 \mathrm{ml}$ de méthanol dans une fiole ambrée de $50 \mathrm{ml}$, puis $15 \mathrm{ml}$ de la solution tampon y ont été ajoutés. Sous une hotte, $179 \mu \mathrm{l}$ de 2-mercaptoéthanol ont été ajoutés à la solution d'OPA. Cette solution de dérivation a été conservée à $24^{\circ} \mathrm{C}$ à l'abri de la lumière. Une semaine après sa préparation, la solution de dérivation n'est plus utilisable.

\section{Principe et procédure de la dérivation du glyphosate}

Le glyphosate ou N(phosphonomethyl) glycine (HOOC- $\mathrm{CH}_{2}-\mathrm{NH}-$ $\left.\mathrm{CH}_{2}-\mathrm{PO}_{3} \mathrm{H}_{2}\right)$ donne en présence de l'hypochlorite, la glycine qui est un acide aminé primaire. Le complexe OPA-2mercaptoéthanol se fixe sur la fonction amine de la glycine pour donner l'isoindole détectable à l'UV ou en fluorescence (Jahnke et al., 2015).

La réaction de conversion du glyphosate en glycine a été réalisée par addition de $5 \mu \mathrm{l}$ d'hypochlorite de sodium $(5 \%)$ à la solution de glyphosate puis la solution obtenue a été homogénéisée et chauffée à $36{ }^{\circ} \mathrm{C}$ au bain marie pendant 30 minutes. La dérivation du glyphosate a été réalisée manuellement juste avant de placer le vial dans le rack, en ajoutant au vial la solution de glyphosate ou l'extrait de sol, suivi de la solution de dérivation dans un ratio volumique (1:1).

Temps de rétention et courbe d'étalonnage du glyphosate

Les concentrations de glyphosate (après dérivation) de $0,38 \mu \mathrm{g} / \mathrm{l}$ et $0,38 \mu \mathrm{g} / \mathrm{l}$ ont été préparées pour l'identification du pic du glyphosate. Un blanc a également été préparé en ajoutant à $10 \mathrm{ml}$ d'eau bidistillée, $5 \mu \mathrm{l}$ d'hypochlorite de sodium $(5 \%)$ et la solution de dérivation. Le mélange (eau bidistillée hypochlorite de sodium) et la solution de dérivation ont été pris en volume égal (ratio volumique (1:1)). Le temps de rétention du glyphosate a été déterminé par la comparaison des chromatogrammes des solutions de glyphosate à celui du blanc. Le temps de rétention a été confirmé par une formulation d'herbicide à base de glyphosate (TASMAN $360 \mathrm{~g} / \mathrm{l})$ de la firme phytosanitaire PHYTOTOP. Une concentration de 0,076 $\mu \mathrm{g} / \mathrm{l}$ du produit commercial a donné après dérivation une concentration finale de 0,038 $\mu \mathrm{g} / \mathrm{l}$, puis cette concentration a été injectée dans le système chromatographique pour la confirmation du temps de rétention. Une fois le temps de rétention déterminé, l'étalonnage du glyphosate a été réalisé avec les solutions étalons (solutions dérivées) de concentrations 0,038, 0,38, 3,8 et 7,6 $\mu \mathrm{g} / \mathrm{l}$.

\section{Conditions chromatographiques}

Les conditions chromatographiques qui ont permis l'identification et la quantification du glyphosate sont les suivantes :

Type de colonne : Shim pack VP-ODS (250 L x 4,6 mm)

Volume d'injection : $10 \mu \mathrm{l}$

Débit : $0,5 \mathrm{ml} / \mathrm{min}$

Eluant A : $50 \%$ acétonitrile 
Eluant B : $50 \% \mathrm{H}_{2} \mathrm{O}+0,5 \%$ de $\mathrm{H}_{3} \mathrm{PO}_{4}$

Mode d'élution : isocratique

Longueur d'onde $(\lambda): 205 \mathrm{~nm}$

\section{Validation de la méthode}

La validation de la méthode a été faite en déterminant les paramètres tels que la linéarité, la fidélité, la limite de détection (LD), la limite de quantification (LQ) et l'exactitude (Résolution Oeno, 2005).

\section{Linéarité}

La linéarité de la gamme d'étalonnage a été testée par une régression linéaire au moyen du logiciel de statistique XLSTAT version 7.5. Les concentrations étalons utilisées sont $0,076,0,76,7,6$ et 15,2 $\mu \mathrm{g} / \mathrm{l}$.

\section{Fidélité}

Pour apprécier la fidélité de la méthode, la répétabilité et la reproductibilité ont été calculées. Lors de la réalisation de la répétabilité, la solution de glyphosate de 15,2 $\mu \mathrm{g} / \mathrm{l}$ a été dérivée pour donner une concentration finale de 7,6 $\mu \mathrm{g} / \mathrm{l}$. Cette concentration a été injectée 10 fois successivement le même jour puis les concentrations données par le HPLC et les surfaces correspondantes ont été relevées.

En ce qui concerne la reproductibilité, la solution de glyphosate de $0,76 \mu \mathrm{g} / \mathrm{l}$ a été dérivée pour donner une concentration finale de $0,38 \mu \mathrm{g} / \mathrm{l}$. L'opération a été effectuée sur 5 jours en raison de 2 injections par jour. Ainsi, 10 injections ont été effectuées sur l'ensemble des 5 jours.

Le coefficient de variation (CV) de chaque paramètre a été calculé selon

$$
\text { 1'expression : } \quad \mathrm{CV}=100 \times\left(\frac{\sigma_{n_{i}-1}}{\bar{x}}\right)
$$

(INRS, 2005).

Avec CV : coefficient de variation

$$
\begin{gathered}
\sigma_{n-1}: \text { écart type } \\
\bar{x}: \text { moyenne }
\end{gathered}
$$

\section{Limite de détection / limite de quantification}

Dans la présente étude, la limite de détection (LD) a été assimilée à la concentration équivalant à 3 fois l'écart type de la plus faible concentration d'étalon, tandis que la limite de quantification a été déterminée en multipliant l'écart type par un facteur de 10 (INRS, 2005 ; Résolution Oeno, 2005). L'écart type lui-même a été déterminé sur 10 injections successives d'une solution dérivée de concentration $0,038 \mu \mathrm{g} / \mathrm{l}$, puis l'écart type de cette série a été calculé suivant l'expression (INRS, 2005) :

$$
\sigma_{n-1}=\sqrt{\frac{\sum_{1}^{n}\left(x_{i}-\bar{x}\right)^{2}}{n-1}}
$$

Avec $X_{i}:$ i $^{\mathrm{e}}$ valeur, obtenue sur une série de n mesures $\bar{\chi}$ : valeur moyenne sur la série des mesures $n$ : nombre de mesures

\section{Exactitude}

L'évaluation de l'exactitude a consisté à doper un échantillon (initialement non contaminé) avec une substance (glyphosate) de concentration et de volume connus, puis à extraire la substance de dopage afin et de calculer le taux de récupération.

Pour se faire, $25 \mathrm{~g}$ de sol séché, ont été contaminés par $1 \mathrm{ml}$ d'une solution de glyphosate à 7,6 $\mu \mathrm{g} / \mathrm{l}$. Le volume de l'extrait a été réduit à $10 \mathrm{ml}$; ce qui a donné la concentration de glyphosate de $0,76 \mu \mathrm{g} / \mathrm{l}$. Un aliquote de $300 \mu \mathrm{l}$ d'extrait concentré, a été dérivé avec $300 \mu \mathrm{l}$ de la solution de dérivation; cela a abouti à une nouvelle concentration de glyphosate de 0,38 $\mu \mathrm{g} / \mathrm{l}$ (concentration théorique). La concentration de cet aliquote a été déterminée par HLPC pour connaître la concentration réelle. L'opération ci-dessus a été réalisée 10 fois afin d'avoir une concentration moyenne de glyphosate. Le taux de récupération a été calculé par l'expression suivante :

Taux de récupération $\quad(\%)=\frac{C_{R}}{C_{T h}} \times 100$ Avec $C_{R}$ : concentration réelle déterminée par le HPLC

$C_{T h}$ : concentration théorique 


\section{Analyses statistiques}

Les statistiques descriptives et les analyses statistiques ont été réalisées au moyen du logiciel de statistique XLSTAT version 7.5. La linéarité de la gamme d'étalonnage du glyphosate a été vérifiée au moyen d'une analyse de régression linéaire simple où le test $\mathrm{F}$ de Fisher a été utilisé avec un seuil de significativité de $5 \%$.

\section{RESULTATS}

\section{Propriétés physico-chimiques du sol}

Les résultats des propriétés physicochimiques des 30 échantillons sont consignés dans le Tableau 1. Les statistiques descriptives et la distribution des paramètres physicochimiques du sol sont mises en évidence par les boîtes à moustaches des Figures 1 et 2 .

La teneur en matière organique du sol se situe entre $5,42 \%$ et $11,8 \%$ avec une teneur moyenne de $8 \%$. Le pH du sol varie entre 3,99 et 7,07 avec une moyenne de pH égale 5,7.

La conductivité du sol se situe entre 0,06 et $0,3 \mathrm{mS} / \mathrm{cm}$ avec une moyenne de 0,116 $\mathrm{mS} / \mathrm{cm}$. La fraction fine du sol est dominée par le sable car la proportion de sable est comprise entre 92,54 et $99,2 \%$, avec une moyenne de 96,03\% tandis que les argiles et les limons ne représentent que $3,97 \%$ de cette fraction fine.

\section{Validation de la méthode de dosage du glyphosate \\ Temps de rétention}

La comparaison des chromatogrammes des solutions étalons de glyphosate $(3,8$ et $0,38 \mu \mathrm{g} / \mathrm{l})$ au chromatogramme du blanc (OPA-2MCE- $\mathrm{H}_{2} \mathrm{O}$ ) a permis d'identifier le pic du glyphosate à 4,568 min avec une précision de $5 \%$. Le chromatogramme du blanc ne donne pas de pic à ce temps (Figures 3-5). En outre, ce temps a été confirmé par le produit technique (formulation d'herbicide à base de glyphosate) pour lequel le pic est apparu à 4,567 min (Figure 7), temps qui est sensiblement identique à celui des solutions de glyphosate.

\section{Linéarité}

Les étalons de glyphosate ont donné les surfaces de pic consignés dans le Tableau 2. La régression linéaire entre les concentrations des étalons et les surfaces de pic, a donné les coefficients d'ajustement du Tableau 3. Le coefficient de détermination $\left(\mathrm{R}^{2}\right)$ de cette régression est 0,9987. En outre, l'évaluation de la quantité d'informations apportée par la variable explicative (surface de pic) au modèle, à travers le test $\mathrm{F}$ de Fisher a donné 0,001 comme valeur de probabilité (Tableau 4). La probabilité associée au test $\mathrm{F}$ de Fisher $(0,001)$ étant $<5 \%$ (seuil de tolérance), on conclut que la surface du pic apporte une quantité d'informations significative au modèle. L'équation du modèle est $\mathrm{Y}=$ $0,0002 \mathrm{X}+0,0027$ et sa représentation graphique est donnée par la Figure 8.

\section{Fidélité de la méthode}

L'injection en dix reprises d'une concentration de glyphosate de 7,6 $\mu \mathrm{g} / \mathrm{l}$ dans le système chromatographique, a donné une concentration moyenne de 7,554 $\mu \mathrm{g} / \mathrm{l}$ avec un coefficient de variation de 0,011 soit $1,1 \%$ (Tableau 5).

Quant à l'évaluation de la reproductibilité, la concentration moyenne de glyphosate de 0,366 $\mu \mathrm{g} / \mathrm{l}$ a été obtenue en utilisant la concentration de $0,38 \mu \mathrm{g} / \mathrm{l}$. L'écarttype et le coefficient de variation de cette opération sont respectivement $0,01 \mu \mathrm{g} / \mathrm{l}$ et 2,9\% (Tableau 6).

\section{Limite de détection, limite de quantification et exactitude}

La limite de détection (LD) est de $0,009 \mathrm{mg} / \mathrm{kg}$ et la limite de quantification (LQ) qui vaut 10 fois l'écart type est de 0,027 $\mathrm{mg} / \mathrm{kg}$.

Pour le taux de récupération, l'injection en dix reprises de la solution de glyphosate à $0,38 \mu \mathrm{g} / \mathrm{l}$, a donné une concentration moyenne de glyphosate de $0,335 \mu \mathrm{g} / \mathrm{l}$ soit un taux de recouvrement de $88,16 \%$. 
Tableau 1 : Paramètres physico-chimiques du sol.

\begin{tabular}{|c|c|c|c|c|c|}
\hline $\mathbf{N}^{\circ}$ & MO & pH & Conductivité & & anulométrie \\
\hline Ech. & $(\%)$ & eau $\left(25^{\circ} \mathrm{C}\right)$ & & \% sable & $\%$ (argile+ limon) \\
\hline 1 & $9,22 \pm$ & $5,18 \pm 0,33$ & $0,14 \pm 0,03$ & $98,20 \pm 2,05$ & $1,8 \pm 0,26$ \\
\hline 2 & $8,52 \pm$ & $6,00 \pm 0,23$ & $0,07 \pm 0,02$ & $93,82 \pm 2,01$ & $6,18 \pm 0,88$ \\
\hline 3 & $11,8 \pm$ & $6,14 \pm 0,18$ & $0,08 \pm 0,02$ & $97,14 \pm 1,05$ & $2,86 \pm 0,23$ \\
\hline 4 & $7,66 \pm$ & $5,97 \pm 0,13$ & $0,12 \pm 0,05$ & $93,57 \pm 1,54$ & $6,43 \pm 0,79$ \\
\hline 5 & $7,30 \pm$ & $5,58 \pm 0,36$ & $0,15 \pm 0,04$ & $98,01 \pm 2,00$ & $1,99 \pm 0,06$ \\
\hline 6 & $6,75 \pm$ & $5,67 \pm 0,24$ & $0,08 \pm 0,02$ & $92,54 \pm 1,65$ & $7,46 \pm 2,05$ \\
\hline 7 & $8,80 \pm$ & $5,83 \pm 0,21$ & $0,08 \pm 0,03$ & $98,42 \pm 1,25$ & $1,58 \pm 0,25$ \\
\hline 8 & $9,56 \pm$ & $4,62 \pm 0,17$ & $0,10 \pm 0,02$ & $95,53 \pm 1,15$ & $4,47 \pm 0,04$ \\
\hline 9 & $7,14 \pm$ & $5,11 \pm 0,22$ & $0,14 \pm 0,04$ & $95,90 \pm 2,21$ & $4,10 \pm 0,07$ \\
\hline 10 & $6,84 \pm$ & $6,60 \pm 0,35$ & $0,18 \pm 0,02$ & $98,20 \pm 3,00$ & $1,80 \pm 2,05$ \\
\hline 11 & $9,12 \pm$ & $6,92 \pm 0,20$ & $0,11 \pm 0,03$ & $92,54 \pm 2,17$ & $7,46 \pm 1,01$ \\
\hline 12 & $8,10 \pm$ & $6,75 \pm 0,27$ & $0,08 \pm 0,02$ & $98,40 \pm 2,41$ & $1,6 \pm 0,08$ \\
\hline 13 & $10,28 \pm$ & $6,24 \pm 0,31$ & $0,18 \pm 0,04$ & $95,52 \pm 3,01$ & $4,48 \pm 0,93$ \\
\hline 14 & $8,80 \pm$ & $7,07 \pm 0,18$ & $0,30 \pm 0,05$ & $95,80 \pm 3,05$ & $4,2 \pm 0,65$ \\
\hline 15 & $6,64 \pm$ & $6,48 \pm 0,13$ & $0,12 \pm 0,02$ & $96,24 \pm 1,57$ & $3,76 \pm 0,85$ \\
\hline 16 & $6,96 \pm$ & $6,88 \pm 0,22$ & $0,08 \pm 0,02$ & $98,20 \pm 1,35$ & $1,8 \pm 0,07$ \\
\hline 17 & $6,62 \pm$ & $5,18 \pm 0,23$ & $0,11 \pm 0,03$ & $93,82 \pm 2,24$ & $6,18 \pm 1,00$ \\
\hline 18 & $6,62 \pm$ & $5,87 \pm 0,14$ & $0,11 \pm 0,04$ & $97,14 \pm 3,15$ & $2,86 \pm 0,51$ \\
\hline 19 & $5,42 \pm$ & $6,39 \pm 0,24$ & $0,07 \pm 0,02$ & $93,57 \pm 1,19$ & $6,43 \pm 0,95$ \\
\hline 20 & $8,51 \pm$ & $6,02 \pm 0,18$ & $0,06 \pm 0,02$ & $98,01 \pm 2,50$ & $1,99 \pm 0,06$ \\
\hline 21 & $6,40 \pm$ & $6,38 \pm 0,19$ & $0,07 \pm 0,03$ & $92,54 \pm 1,04$ & $7,46 \pm 0,97$ \\
\hline 22 & $6,75 \pm$ & $6,79 \pm 0,27$ & $0,14 \pm 0,04$ & $98,42 \pm 2,10$ & $1,58 \pm 0,07$ \\
\hline 23 & $7,56 \pm$ & $5,32 \pm 0,22$ & $0,15 \pm 0,05$ & $95,53 \pm 1,01$ & $4,47 \pm 1,02$ \\
\hline 24 & $8,84 \pm$ & $5,37 \pm 0,31$ & $0,13 \pm 0,02$ & $95,90 \pm 2,02$ & $4,1 \pm 0,91$ \\
\hline 25 & $8,86 \pm$ & $5,21 \pm 0,20$ & $0,09 \pm 0,03$ & $96,14 \pm 3,01$ & $3,86 \pm 0,52$ \\
\hline 26 & $6,75 \pm$ & $5,19 \pm 0,26$ & $0,09 \pm 0,04$ & $99,20 \pm 2,05$ & $0,8 \pm 0,05$ \\
\hline 27 & $7,56 \pm$ & $4,51 \pm 0,12$ & $0,07 \pm 0,02$ & $93,82 \pm 1,20$ & $6,18 \pm 1,05$ \\
\hline 28 & $8,84 \pm$ & $4,37 \pm 0,28$ & $0,13 \pm 0,03$ & $97,14 \pm 2,25$ & $2,86 \pm 0,81$ \\
\hline 29 & $8,86 \pm$ & $3,99 \pm 0,19$ & $0,13 \pm 0,02$ & $93,57 \pm 1,15$ & $6,43 \pm 0,75$ \\
\hline 30 & $9,08 \pm$ & $4,05 \pm 0,16$ & $0,12 \pm 0,04$ & $98,01 \pm 3,02$ & $1,99 \pm 0,07$ \\
\hline
\end{tabular}

$\mathrm{N}^{\circ}$ Ech.= Numéro de l'échantillon, $\mathrm{MO}=$ Matière Organique

Tableau 2 : Surface de pic en fonction de la concentration du glyphosate.

\begin{tabular}{cc}
\hline Surface & Concentration $(\boldsymbol{\mu g} / \mathbf{l})$ \\
\hline 365 & 0,038 \\
1940 & 0,38 \\
16059 & 3,8 \\
34210 & 7,6 \\
\hline
\end{tabular}


G. K. KPAN KPAN et al. / Int. J. Biol. Chem. Sci. 9(3): 1384-1398, 2015

Tableau 3 : Coefficients d'ajustement de la régression linéaire entre les concentrations étalons et les surfaces de pic associées.

\begin{tabular}{ll}
\hline Paramètres & Valeur \\
\hline $\mathrm{R}$ (coefficient de corrélation) & 0,9993 \\
$\mathrm{R}^{2}$ (coefficient de détermination) & 0,9987 \\
$\mathrm{R}^{2}$ aj. (coefficient de détermination ajusté) & 0,9980 \\
$\mathrm{SCR}$ & 0,0493 \\
\hline \multicolumn{1}{c}{$\mathrm{SCR}=$ Somme des Carrés Résiduelles } &
\end{tabular}

Tableau 4 : Résultat du test $\mathrm{F}$ réalisé pour la régression linéaire.

\begin{tabular}{cccccc}
\hline Source & ddl & Somme des carrés & $\begin{array}{c}\text { Carré } \\
\text { moyen }\end{array}$ & $\begin{array}{c}\mathbf{F} \\
\text { de Fisher }\end{array}$ & Pr > F \\
\hline Modèle & 1 & 37,380 & 37,380 & 1516,305 & 0,001 \\
Résidus & 2 & 0,049 & 0,025 & & \\
Total & 3 & 37,430 & & & \\
\hline \multicolumn{5}{r}{ ddl = degré de liberté, $P r$ = Probabilité }
\end{tabular}

Tableau 5 : Statistiques descriptives de la répétabilité.

\begin{tabular}{ccccc}
\hline $\begin{array}{c}\mathbf{N}^{\circ} \\
\text { injection }\end{array}$ & $\begin{array}{c}\text { Concentration } \\
(\boldsymbol{\mu g} / \mathbf{l})\end{array}$ & $\begin{array}{c}\text { Moyenne } \\
(\boldsymbol{\mu g} / \mathbf{l})\end{array}$ & $\begin{array}{c}\text { Ecart- type } \\
(\boldsymbol{\mu g} / \mathbf{l})\end{array}$ & $\begin{array}{c}\text { Coefficient de } \\
\text { Variation CV }(\%)\end{array}$ \\
\hline 1 & 7,5823 & & & \\
2 & 7,7113 & & & \\
3 & 7,5811 & & & \\
4 & 7,5713 & 7,554 & 0,080 & \\
5 & 7,5609 & & & \\
6 & 7,4709 & & \\
7 & 7,5393 & & \\
8 & 7,5821 & & \\
9 & 7,4213 & & \\
10 & 7,5465 & & \\
\hline
\end{tabular}

Tableau 6 : Statistiques descriptives de la reproductibilité.

\begin{tabular}{ccccc}
\hline $\begin{array}{c}\mathbf{N}^{\circ} \\
\text { injection }\end{array}$ & $\begin{array}{c}\text { Concentration } \\
(\boldsymbol{\mu} \mathrm{g} / \mathbf{l})\end{array}$ & $\begin{array}{c}\text { Moyenne } \\
(\boldsymbol{\mu g} / \mathbf{l})\end{array}$ & $\begin{array}{c}\text { Ecart- type } \\
(\boldsymbol{\mu g} / \mathbf{l})\end{array}$ & $\begin{array}{c}\text { Coefficient de } \\
\text { Variation CV }(\%)\end{array}$ \\
\hline 1 & 0,3907 & & & \\
2 & 0,3707 & & & \\
3 & 0,3571 & & & \\
4 & 0,3599 & 0,366 & & \\
5 & 0,3597 & & & \\
6 & 0,3707 & & \\
7 & 0,3635 & & & \\
8 & 0,3707 & & \\
9 & 0,3557 & & \\
10 & 0,3587 & & \\
\hline
\end{tabular}


G. K. KPAN KPAN et al. / Int. J. Biol. Chem. Sci. 9(3): 1384-1398, 2015

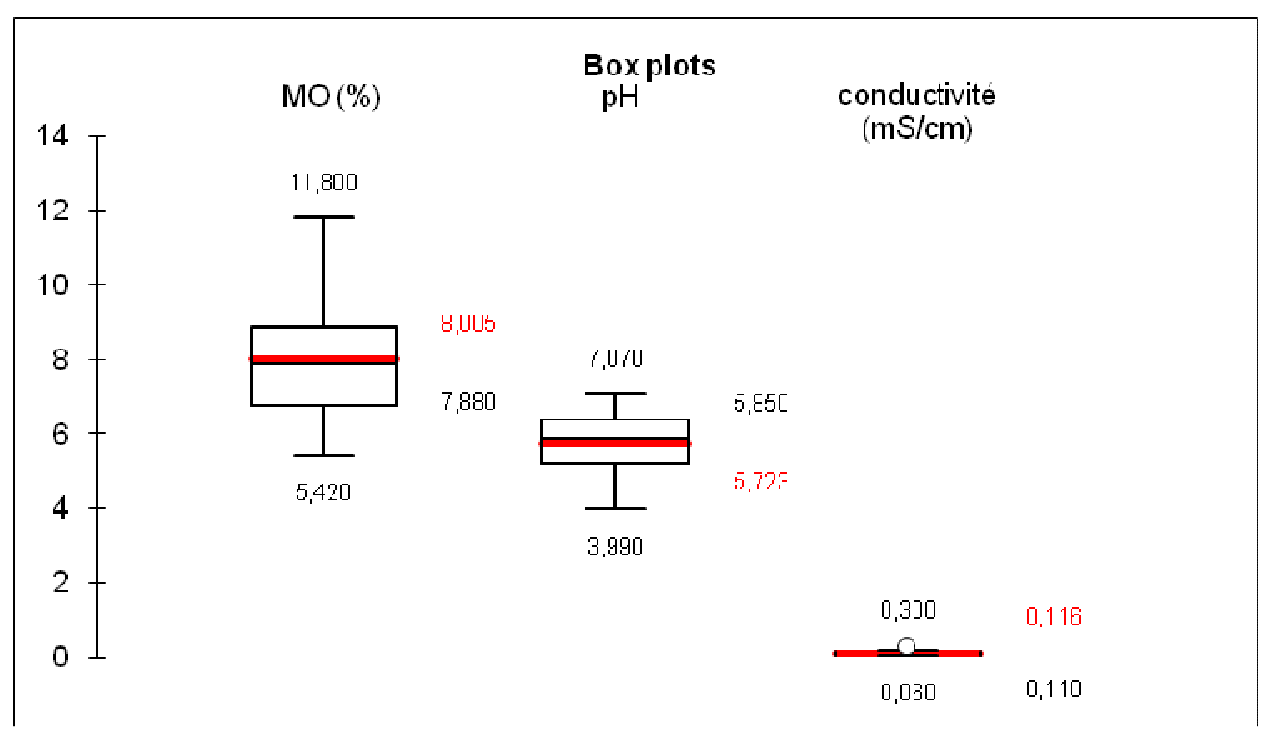

Figure 1 : Distribution des paramètres physico-chimiques : matière organique (MO), pH et conductivité du sol.

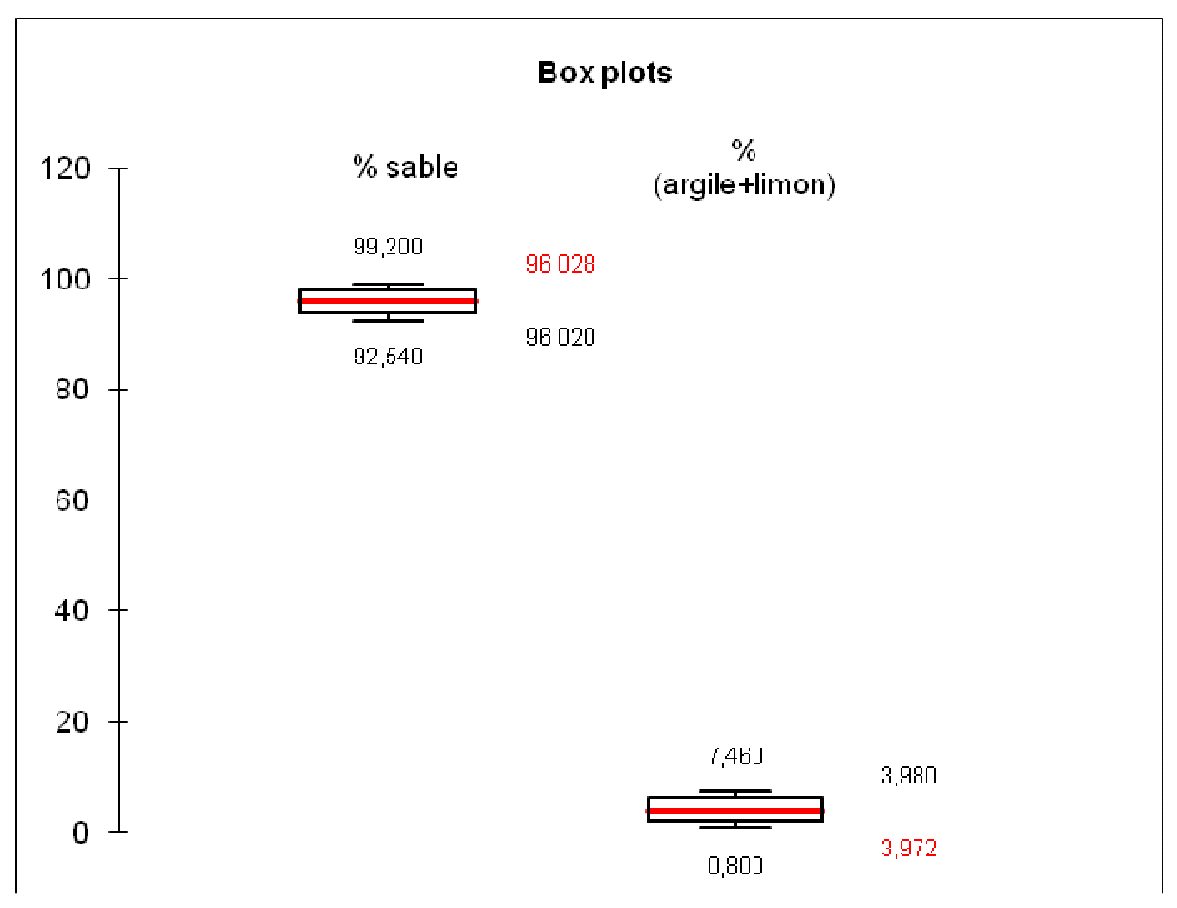

Figure 2 : Statistiques descriptives de la granulométrie. 
G. K. KPAN KPAN et al. / Int. J. Biol. Chem. Sci. 9(3): 1384-1398, 2015

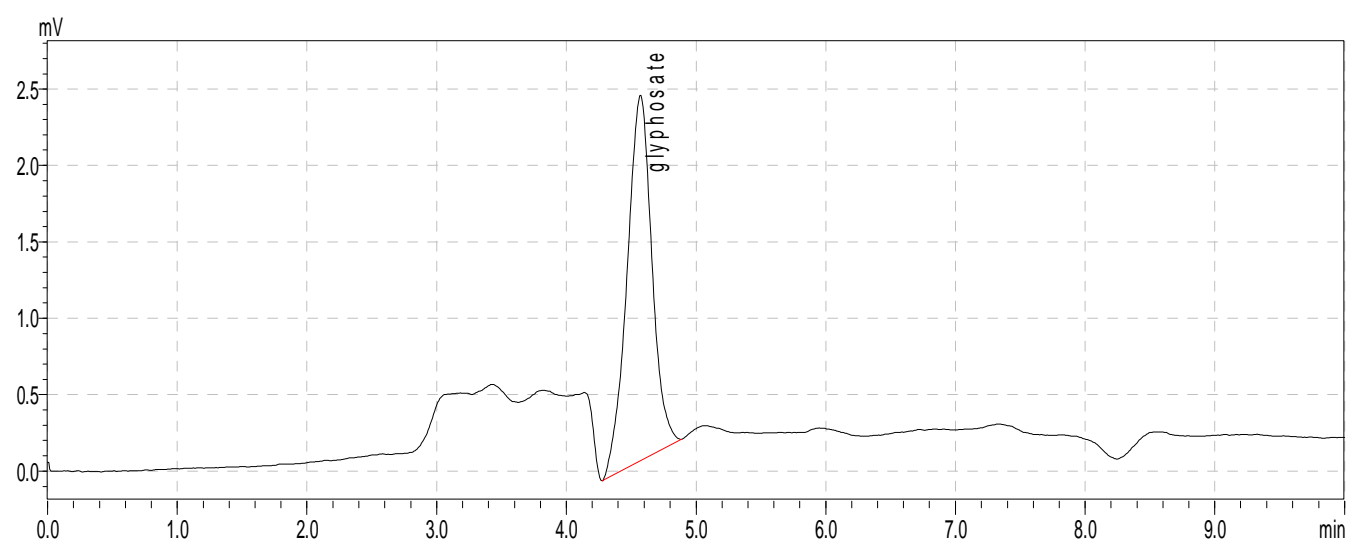

Figure 3 : Chromatogramme d'un étalon de glyphosate à 3,8 $\mu \mathrm{g} / \mathrm{l}$.

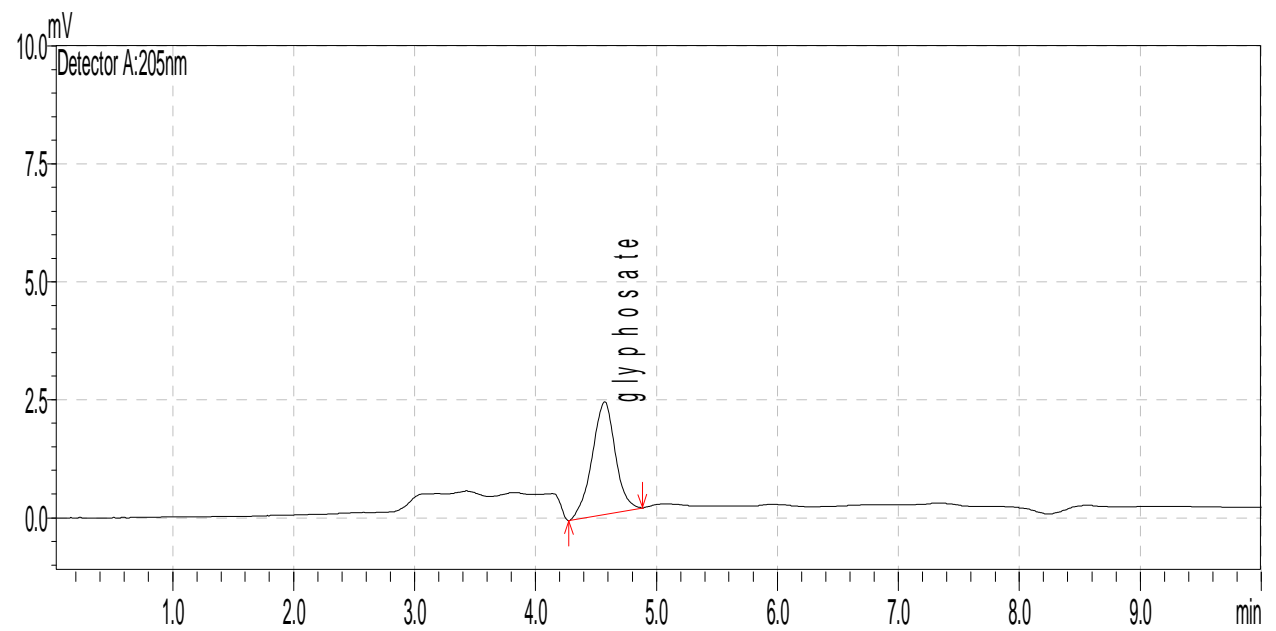

Figure 4 : Chromatogramme d'un étalon de glyphosate à $0,38 \mu \mathrm{g} / \mathrm{l}$.

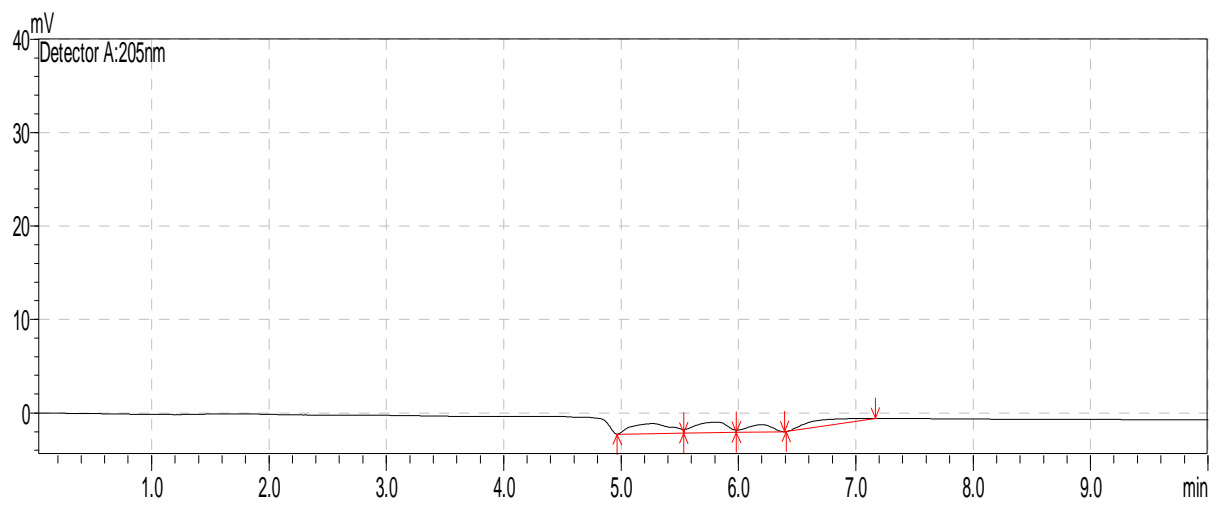

Figure 5 : Chromatogramme du blanc (eau-OPA-2MCE). 


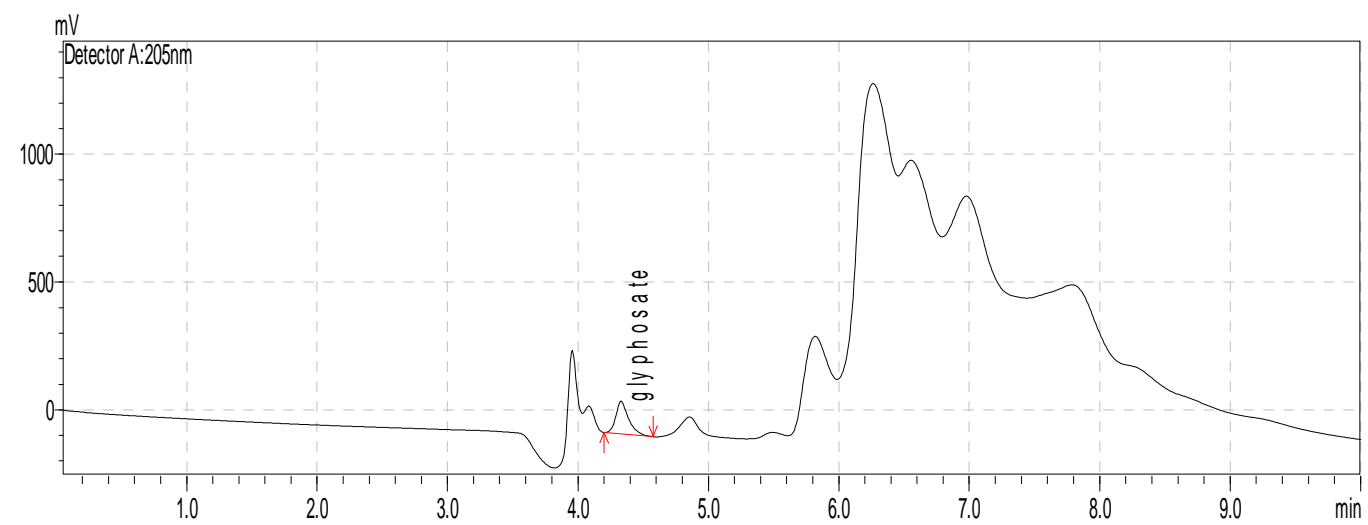

Figure 7 : Chromatogramme d'une formulation d'herbicide (TASMAN 360g/l de glyphosate) à $0,038 \mu \mathrm{g} / \mathrm{l}$.

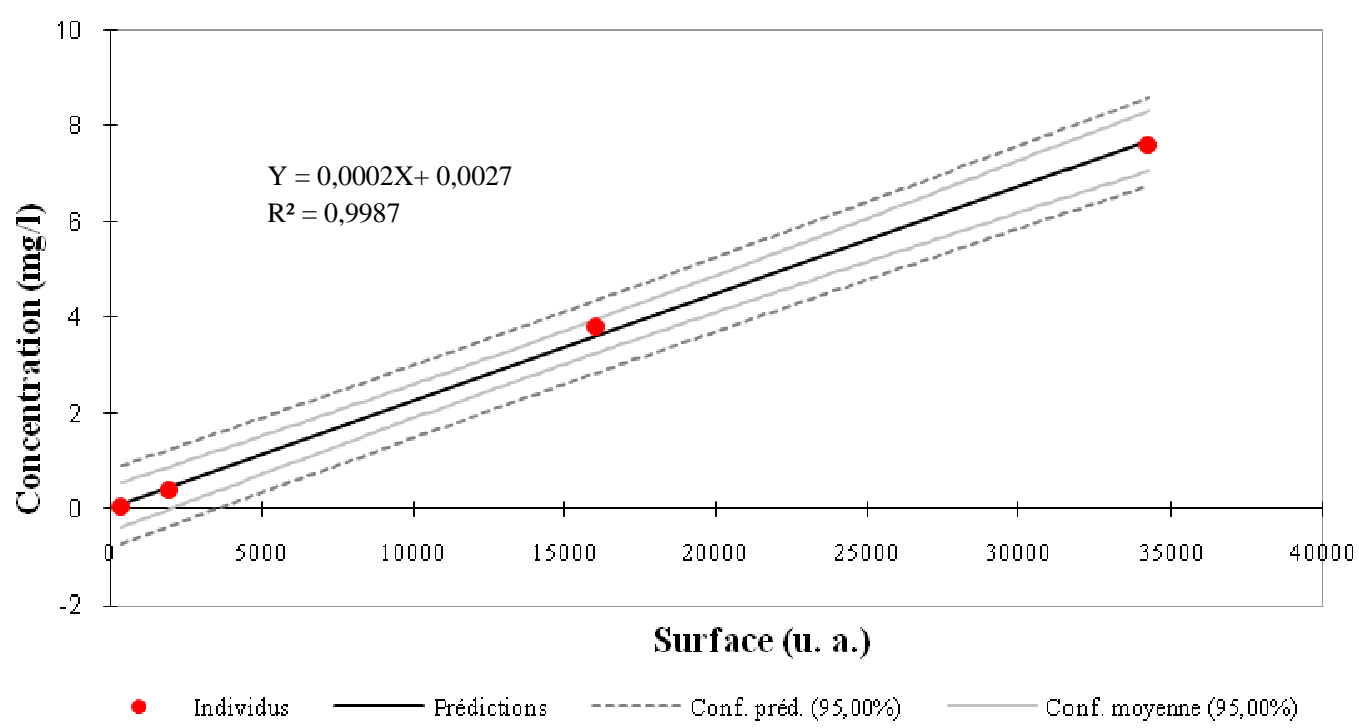

Figure 8 : Droite de régression linéaire entre les concentrations de glyphosate et les surfaces de pic associées.

\section{DISCUSSION}

\section{Paramètres physico-chimiques}

Les paramètres physico-chimiques sont moins dispersés dans la mesure où les boîtes à moustaches sont de petites tailles (Le Guen, 2001). Cela traduit une moindre fluctuation des mesures réalisées.

La teneur moyenne en matière organique du sol (8\%) montre qu'il s'agit d'un sol très pauvre en matière organique (Zro Bi et al., 2012). Cependant, cette teneur en matière organique est relativement élevée par rapport à celles signalées par Koko et al. (2008) dans les sols du Sud-Ouest de la Côte d'Ivoire. En effet, ces auteurs ont trouvé des pourcentages de matières organiques $<4 \%$ dans les sols sous cacaoyers. Cette différence de teneur en matière organique pourrait s'expliquer par la perte en matière organique due à l'exploitation agronomique des sols 
sous cacaoyers tandis que le sol d'Azaguié est au repos depuis environ 25 ans.

La valeur moyenne du $\mathrm{pH}$ du sol $(5,7)$, atteste que ce sol est acide (Zro Bi et al., 2012). Cette moyenne de $\mathrm{pH}$ est plus élevée que les valeurs de $\mathrm{pH}(4,7$ - 5) obtenues par Kabrah et al. (2000) pour les sols sous palmeraies de la région de la Mé (Côte d'Ivoire).

Quant à la conductivité, les valeurs de la présente étude sont faibles rapport à celles enregistrées par Sene et al. (2014) dans les sols de la vallée rizicole de Tamra. En effet, ces auteurs ont mesuré des valeurs conductivité oscillant entre 12,4 et 0,04 $\mathrm{mS} / \mathrm{cm}$ alors que dans la présente étude la conductivité du sol se situe entre 0,06 et 0,3 $\mathrm{mS} / \mathrm{cm}$. Cette différence de conductivité pourrait être due au fait que les sols de Tamra qui sont des sols de vallée, reçoivent les sels minéraux érodés des zones alentours ; ce qui augmente leur salinité tandis que le sol d'Azaguié qui est un sol en pente perd constamment des minéraux sous l'effet de l'érosion; par conséquent, ce sol a une faible salinité

\section{Validation de la méthode de dosage du glyphosate}

Le temps de rétention du glyphosate (4,568 $\mathrm{min})$ est un temps relativement court, comparé à celui de 7,5 min obtenu par Eduardo et al. (2014). Un tel temps est très bénéfique dans la mesure où il permet un gain du temps et une économie de solvant. Cependant, il faut signaler que la méthode de détermination du glyphosate par HPLC de Gouli Bi et al. (2011) a un temps de rétention (1,6 min) plus court que celui de la présente méthode. Cette différence entre les temps de rétention serait due aux paramètres analytiques tels que la composition des solutions d'élution, le débit d'élution et les longueurs d'onde d'adsorption utilisés les différents travaux. En effet, Gouli Bi et al. (2011) ont dérivé le glyphosate avec du triméthylamine / phenylisothiocyanate et ont détecté le complexe obtenu à 254 nm, avec une élution isocratique de $1 \mathrm{ml} / \mathrm{min}$. Quant à la méthode d'Eduardo et al. (2014), le glyphosate a été dérivée à l'aide du chlorure de p-toluenesulfonyl et la détection a été effectuée à $240 \mathrm{~nm}$.

Le coefficient de détermination $\left(\mathrm{R}^{2}\right)$ de 0,9987 signifie que $99,87 \%$ de la variabilité de la concentration de glyphosate sont expliqués par la surface du pic. Il s'agit donc d'un bon coefficient de détermination dans la mesure où il est largement supérieur $50 \%$ et très proche de 1 . Aussi, ce coefficient est-t-il sensiblement égal au coefficient de détermination de méthode de dosage du glyphosate par HPLC développée par Gouli Bi et al. (2011) dont le ( $\left.\mathrm{R}^{2}\right)$ est de 0,998.

Les coefficients de variation de la répétabilité $(1,1 \%)$ et de la reproductibilité $(2,9 \%)$ sont acceptables car ils sont endessous de $5 \%$. Ces coefficients de variation sont nettement inférieurs à ceux obtenus par Le Fur et al. (2000) lors du dosage du glyphosate dans l'eau de surface, lesquels coefficients oscillent entre $6,49 \%$ et $17,12 \%$.

La limite de quantification de la méthode est acceptable car elle est inférieure à la plupart des Limites Maximales des Résidus (LMR) du glyphosate tant pour les sédiments que pour les denrées alimentaires. En effet, au niveau du Codex Alimentarius, la plus faible LMR du glyphosate est de $0,05 \mathrm{mg} / \mathrm{kg}$ et correspond aux denrées telles que la banane, la viande et le lait (Codex Alimentarius, 2013). Au niveau de l'Agence Américaine pour la Protection Environnementale (USEPA), la plus faible LMR du glyphosate est de $0,1 \mathrm{mg} / \mathrm{kg}$ et correspond au coco et aux graines de crambe (US-EPA, 2012).

Quant aux sédiments, la plus faible valeur guide de LMR proposée par l'Institut National de l'Environnement Industriel et des Risques (INERIS) est de $0,5 \mathrm{mg} / \mathrm{kg}$ (INERIS, 2014). Par ailleurs, pour les méthodes de détermination du glyphosate dans le sol, la limite de quantification de la présente méthode $(0,027 \mathrm{mg} / \mathrm{kg})$ est nettement inférieure à la LQ $(0,10 \mathrm{mg} / \mathrm{kg})$ trouvée par Peruzzo et al. (2008). Toutefois, la LQ (0,027 
$\mathrm{mg} / \mathrm{kg})$ est plus élevée que la LQ $(0,006 \mu \mathrm{g} / \mathrm{g})$ $\mathrm{du}$ glyphosate obtenue par Börjesson et Torstensson (2000) lors de la détermination du glyphosate dans le sol.

Le taux de récupération de la présente méthode $(88,16 \%)$ est acceptable car elle est au-dessus de $50 \%$. Ce taux de récurrence est dans la gamme des taux de recouvrement de la plupart des méthodes de détermination du glyphosate du sol et des denrées alimentaires ; c'est le cas de Peruzzo et al. (2008) et de Nedelkoska et Low (2004) qui ont trouvé les taux de recouvrement respectifs de $96 \%$ et de $82,4 \%$.

\section{Conclusion}

La méthode de dosage du glyphosate mise au point dans la présente étude est une méthode quasi complète car elle part de l'extraction à la quantification de la molécule en passant par l'étape de la dérivation. Il s'agit d'une méthode relativement réalisable, car les solvants et réactifs pris en compte par la méthode sont tous accessibles. A l'instar des autres méthodes de détermination du glyphosate dans le sol, la présente méthode mérite une attention particulière dans la mesure où la LQ $(0,027 \mathrm{mg} / \mathrm{kg})$ est inférieure aux plus sévères LMR du glyphosate fixées par le Codex Alimentarius et l'EPA. En outre, le taux de recouvrement $(88,16 \%)$ est élevé et le temps de rétention $(4,567 \mathrm{~min})$ est assez court. Par ailleurs, cette méthode est reproductible avec un coefficient de variation de $2,9 \%$.

Cependant, la difficulté majeure rencontrée dans la mise au point de la présente méthode est la dilution. En effet, le glyphosate n'est détectable par cette méthode que lorsqu'il est en faible quantité, cela nécessite plusieurs dilutions qui peuvent provoquer une sous évaluation de la molécule compte tenu des pertes liées à la dilution. Par ailleurs, le taux de recouvrement peut légèrement varier si l'on change de matrice (sol) car les paramètres physico-chimiques $(\mathrm{pH}$, matière organique, conductivité et granulométrie) peuvent influencer la rétention du glyphosate par le sol.

\section{REMERCIEMENTS}

Nous remercions infiniment le Laboratoire Central d'Agrochimie et d'Ecotoxicologie (LCAE) grâce auquel ce travail a pu s'accomplir. Nos remerciements sont vivement adressés au personnel de ce laboratoire pour sa disponibilité et son esprit d'équipe.

\section{REFERENCES}

Börjesson E, Torstensson L. 2000. New methods for determination of glyphosate and (aminomethyl) phosphonic acid in water and soil. Journal of Chromatography A, 886(1-2): 207 - 216.

CEAQ (Centre d'Expertise en Analyse Environnementale du Québec). 2003. Détermination de la matière organique par incinération : méthode de perte au feu (PAF). MA. 1010 - PAF 1.0, Ministère de l'Environnement du Québec, 9p.

CEAQ (Centre d'Expertise en Analyse Environnementale du Québec). 2003. Détermination du $\mathrm{pH}$ à l'eau et du $\mathrm{pH}$ tampon dans les sols agricoles: méthode électrométrique. MA. $205-\mathrm{pH} 1.0$ Ministère de l'Environnement du Québec, 13p.

Chiu HY, Lin ZY, Tu HL, Whang CW. 2008. Analysis of glyphosate and aminomethylphosphonic acid by capillary electrophoresis with electrochemiluminescence detection. Journal of Chromatography A, 1177(1): 195-198.

Codex alimentarius. 2013. Pesticide residues in food and feed, 158 glyphosate. http://www.codexalimentarius.net/pestres /data/pesticides/details.html, consulté le 14/04/2015.

Druart C, Delhomme O, Vaufleuty A, Ntcho E, Millet M. 2011. Optimization of extraction procedure and chromatographic separation of glyphosate, glufosinate and 
aminomethylphosphonic acid in soil. Analytical and Bioanalytical Chemistry, 399(4): 1725-1732.

Duke SO, Powles SB. 2008. Glyphosate: a once-in-a-century herbicide. Pest Management Science, 64: 319-325.

Eduardo LD, Bertozzi J, Evelázio NS, Cláudio CO. 2014. Determination of glyphosate and aminomethylphosphonic acid forassessing the quality taps water using SPE and HPLC. Acta Scientiarum Technology, 36(3): 513-519.

Gouli Bi I, Yapo AJ, Dembélé A, Ello A, Trokourey A. 2011. Determination of glyphosate by high performance liquid chromatography (HPLC) without prior extraction. International Journal of Biological and Chemical Sciences, 5(1): 314-320.

Hasine BH, Aloui T, Gallali T, Bouzid T, El Amri S, Hassen BR. 2008. Evaluation quantitative et rôle de la matière organique dans les sols cultivés en zones subhumides et semi-arides méditerranéenne de la Tunisie. Agrosolutions, 19(2) : 4-17.

INERIS (Institut National de 1'Environnement Industriel et des Risques). 2014. Valeur guide environnementale. Glyphosate $-\mathrm{n}^{\circ}$ CAS : 1071-83-6, INERIS, 22p.

INRS (Institut National de Recherche et de Sécurité). 2005. Calcul de l'incertitude dans les méthodes de mesurage de l'exposition professionnelle. Fiche G/V01, 35p.

Jahnke B, Frank C, Fernández JF, Niemeyer B. 2015. A sequential injection analysis method for the determination of glyphosate and aminomethylphosphonic acid in water samples. American Chemical Science Journal, 5(2): 163-173.

Kabrah Y, Koffi B. 2000. Caractéristiques chimiques du sol sous palmeraie. Cahiers Agricultures, 9(1): 73-76.

Khrolenko MV, Wieczorek PP. 2005. Determination of glyphosate and its metabolite aminomethylphosphonic acid in fruit juices using supported-liquid membrane preconcentration method with high-performance liquid chromatography and UV detection after derivatization with p-toluenesulphonyl chloride. Journal of Chromatography A, 1093(1-2): 111117.

Koko KL, Yoro RG, Ngoran K., Assa A. 2008. Evaluation de la fertilité des sols sous cacaoyers dans le Sud-Ouest de la Côte d'Ivoire. Agronomie Africaine, 20(1): 81- 95.

Le Fur E, Colin R., Charrêteur C, Dufau C, Péron JJ. 2000. Determination of glyphosate herbicide and aminomethylphosphonic acid in natural waters by liquid chromatography using pre-column fluorogenic labeling. Part I: Direct determination at the $0,1 \mu \mathrm{g} / \mathrm{L}$ level using FMOC. Analusis, 28 : 813-818.

Le Guen M. 2001. La boîte à moustaches de TUKEY, un outil pour initier à la Statistique. Statistiquement votre - SFDS, $1-3$.

Li B, Deng X, Guo D, Jin S. 2007. Determination of glyphosate and aminomethylphosphonic acid residues in foods using high performance liquid chromatography-mass spectrometry/mass spectrometry. Chinese Journal of Chromatography, 25(4): 486-490.

Montoroi JP.1997. Conductivité de la solution $\mathrm{du}$ sol et d'extrait aqueux de sol : application à un sol sulfaté acide salé de Basse - Casamance (Sénégal). Etude et Gestion des Sols, 4(4): 279-298.

Nedelkoska TV, Low GKC. 204. Highperformance liquid chromatographic determination of glyphosate in water and plant material after pre-column derivatisation with 9-fluorenylmethyl chloroformate. Analytica Chimica Acta, 511(1): 145-153.

Owen MD, Zelaya IA. 2005. Herbicideresistant crops and weed resistance to herbicides. Pest Management Science, 61: 301-311.

Peruzzo PJ, Porta AA, Ronco AE. 2008. Levels of glyphosate in surface waters, 
sediments and soils associated with direct sowing soybean cultivation in north pampasic region of Argentina. Environmental Pollution, 156: 61 - 66.

Qian KQ, Tang T, Shi T, Wang F, Li J, Cao Y. 2009. Residue Determination of Glyphosate in Environmental Water Samples with High-Performance Liquid Chromatography and UV Detection after Derivatization with 4-Chloro-3,5 dinitrobenzotrifluoride. Analytica Chimica Acta, 635(2): 222-226.

Résolution Oeno. 2005. Recommandations harmonisées pour la validation des méthodes d'analyse par un seul laboratoire. Recueil des méthodes internationales d'analyses - OIV, 18p.

Schronbrunn E, Eschenburg S, Shuttleworth WA, Schloss J. V, Amrhein N, Evans JNS, Kabsch W. 2001. Interaction of the herbicide glyphosate with its target enzyme 5-enolpyruvylshikimate3phosphate synthase in atomic detail. Proceedings of the National Academy of Sciences U.S.A, 98: 1376-1380.

Sene JH B, Matty F, Diatta M. 2014. Caractérisation des sols de la vallée rizicole de Tamra dans l'île de Mar, Centre-Ouest du Sénégal. International Journal of Biological and Chemical Sciences, 8(2): 794-810.

US-EPA (USA Environmental Protection Agency). 2012. Index to pesticide chemical names, part 180 tolerance information, and food and feed commodities (by commodity). 314p.

Zro Bi GF, Yao KA, Kouamé KF.2012. Évaluation statistique et spatiale de la fertilité rizicole des sols hydromorphes (gleysols) de la région du Bélier (Côte d'Ivoire). Tropicultura, 30(4): 236-242. 